Results: For death during the transport and in the emergency department, the receiver-operator curve of two groups reflected as 0.721 and 0.649 . For death in a consequence, the receiveroperator curve of the two groups was revealed as 0.667 and 0.519 .

Discussion: As an accurate triage method, the Sacco Triage Method may be used in a mass casualty incident. It is a more effective way than the START Triage Method for the evaluation of death risk assessment of the mass trauma patients.

Prehosp Disaster Med 2019;34(Suppl. 1):s109-s110

doi:10.1017/S1049023X19002309

\section{Comparison of Traumatic Brain Injury Patients by Age Group in Emergency Department}

Dr. Kwang-Real Huh, A/Prof. Jung-youn Kim, A/Prof. YoungHoon Yoon, Prof. Sung-Woo Moon, Dr. Eu-Sun Lee,

Dr. Bosun Shim

Department of Emergency Medicine, College of Medicine, Korea University, Seoul, South Korea

Introduction: Traumatic brain injury (TBI) is an important public health concern because of the high mortality rate of young people and a high proportion among the trauma. According to studies, patients visiting the emergency department (ED) with TBI comprise $1.4 \%$ of all ED patients.

Aim: The authors think that the characteristics of patients with TBI will vary according to the age group. Therefore, the purpose of this study is to investigate the clinical and social characteristics of patients with TBI visiting the ED by age group. Methods: Trauma patients who conducted brain CT at the ED of Korean University Hospital (three hospitals) for 3 years from March 2013 to February 2016 were enrolled. Medical records were investigated retrospectively. The GCS scores were estimated at initial ED arrival. The primary outcome was to determine the characteristics of each age groups with gender, severity (by GSC score), trauma mechanism, and admission rate.

Results: A total of 15,567 TBI patients received brain CT evaluation during the investigation period. Based on age, patients in their 50s were the most common (16.5\%). Regarding the severity, the ratio of mild was higher in under patients under 9 (99.3\%); the ratio of severe was higher for patients in their $20 \mathrm{~s}(4.6 \%)$. In almost every age group, the male ratio of TBI was higher, except for females aged 70 or older. Under 19 years of age, the ambulance utilization rate was lower than any other age group. The most common injury mechanism was a collision, the next was a traffic accident, and in under 9, a fall was the most common. $70.1 \%$ of patients returned home after treatments.

Discussion: Identifying the characteristics of patients with TBI visiting $\mathrm{ED}$ is fundamental. Therefore, it is necessary to continuously collect basic data on TBI among patients visiting the ED.

Prehosp Disaster Med 2019;34(Suppl. 1):s110

doi:10.1017/S1049023X19002310
A Comparison of Two Types of Personal Protective Equipment (PPE) Doffing Process: Frequency and Sites of Contamination

Mrs. You Jian-ping, Mrs. Yang Sha, Mrs. Luo Hong-Xia, Zhang Hui-Lan

Department of Infectious Diseases, Southwest Hospital, Third Military Medical University, Chongqing, China

Introduction: Personal protective equipment (PPE) is a necessary item in the period of unknown and high-risk emerging infectious disease. It is not only the necessary requirement of strict isolation, but also the last line of defense to protect medical staff.

Aim: Compare the differences between contaminated frequency and sites under two types of PPE doffing.

Methods: Recruited 56 health care workers (HCWs) who worked in clinical to follow the different PPE removal guidelines issued by the Chinese Center for Disease Control (CDC) and the World Health Organization (WHO) final resolution for preventing Ebola virus. Eight batches of HCWs were divided to conduct simulations of contaminated PPE removal using fluorescent lotion (Glitter Bug Potion, On Solution Pty Lt). Then we recorded the frequency and sites of contamination of personnel after removal of contaminated PPE by the method of visual observation.

Results: According to China's CDC process, the parts that are easily contaminated during PPE removal are: left hand and wrist (7 times), left calf (7 times), front chest center and left and right chest (6 times each) and left abdomen (5 times). Contaminated parts of the PPE process in accordance with the $\mathrm{WHO}$ process from high to low were: right hand and wrist (13 times), left hand and wrist (12 times), middle of the abdomen (10 times), left chest (9 times), and left abdomen (6 Times). There was no statistical difference between the two kinds of PPE piercing and removal $(\mathrm{Z}=1.177, \mathrm{P}>0.05)$. Discussion: Under the guidance of the two processes recommended by China CDC and WHO, there was no significant difference in the frequency of pollution after removing PPE. It is speculated that the PPE recommendation processes issued by WHO and China CDC are effective for personal protection against fulminating infectious diseases.

Prehosp Disaster Med 2019;34(Suppl. 1):s110

doi:10.1017/S1049023X19002322

\section{Concerns for Small Hospitals in Rationalising Trauma} Services: How Do We Ensure Enhanced Patient Services in Rural Areas?

Dr. Michael Molloy ${ }^{1,2,3}$, Mr. Paul Kelly ${ }^{2,3}$, A Prof. Gregory

$R$ Ciottone ${ }^{1,4}$

1. BIDMC Fellowship in Disaster Medicine, Boston, United States

2. Wexford General Hospital, Wexford, Ireland

3. University College Dublin, Belfield, Dublin, Ireland

4. Department of Emergency Medicine, Harvard Medical School, Boston, United States

Introduction: Trauma bypass has been introduced successfully worldwide with sustained reductions in mortality/morbidity. Analyzing structure, process, and outcome individually and 